Pacific Journal of Mathematics

OPERATOR VALUED ANALYTIC FUNCTIONS AND
GENERALZATIONS OF SPECTRAL THEORY 


\section{OPERATOR VALUED ANALYTIC FUNCTIONS AND GENERALIZATIONS OF SPECTRAL THEORY}

\section{LOTHROP MitTENTHAL}

This paper is concerned with an analytic operator valued function $F(\lambda)$ acting upon a Banach space $X$, where $F(\lambda)$ is bounded and $F(\lambda) F(\mu)=F(\mu) F(\lambda)$ for all $\lambda, \mu \in \Delta$ where $\Delta$ is the domain of analyticity of $F(\lambda)$. The singular set of $F(\lambda)$ is analogous to the spectrum of a single operator. In the case of the single operator, employing the corresponding resolvent operator, a number of interesting properties are known to be associated with the spectral sets. These include projections and homomorphisms between scalar valued analytic functions and functions of the operator. This paper considers a suitable generalization of the resolvent operator and which properties of spectral sets carry over to open and closed subsets of the singular set of the operator valued analytic function.

It is shown that a suitable generalization of the resolvent operator is $F^{\prime}(\lambda) F(\lambda)^{-1}=F(\lambda)^{-1} F^{\prime}(\lambda)$, from our assumed commutativity, where $F^{\prime}(\lambda)=(d / d \lambda) F(\lambda)$. In addition, it is shown that certain proper open and closed subsets of the singular set, termed separating subsets, have many of the properties of spectral sets. These properties include a relation between ascent and descent of the operator and the order of the pole of the generalized resolvent, projections analogous to spectral projections, and an operational calculus. A sufficient condition for a singular subset to be separating is derived. In addition, a new operator is defined which in a sense represents the $F(\lambda)$ on the subspace corresponding to a given separating singular subset.

Definition. The singular set of $F(\lambda), S(F(\lambda))$ or $S(F)$, is the set of all $\lambda \in \mathscr{C}$, the complex plane, such that $F(\lambda)$ is not continuously invertible. The complement of $S(F)$ will be called the regular set, $R(F(\lambda))$ or $R(F)$.

In this paper, it will be assumed that $S(F)$ is bounded and that $S(F) \subset \Delta$. In particular, this will include polynomials with operator coefficients, e.g. $F(\lambda)=\lambda^{n} I+\lambda^{n-1} A_{1}+\cdots+A_{n}$ where the $A_{j}$ are all bounded commuting operators. It has been shown by A. Taylor [3, p. 590] that on $R(F) \cap \Delta, F(\lambda)^{-1}$ is also analytic. As in the case of the spectrum of a single operator, it is easy to show that $R(F)$ is open and that $S(F)$ is closed.

If, for example, we choose $F(\lambda)=\lambda^{n} I+\lambda^{n-1} A_{1}+\cdots+A_{n}$ and 
let $D$ be a bounded Cauchy domain such that $S(F) \subset D$, then an easy calculation gives

$$
\frac{1}{2 \pi i} \oint_{\partial D} F^{\prime}(\lambda) F(\lambda)^{-1} d \lambda=n I
$$

which is not a projection for $n>1$ and by analogy with analytic function theory, $n$ in a sense represents the number of "zeroes" of $F(\lambda)$. For the above polynomial, Equation (1) holds even if the coefficient operators $A_{j}$ do not commute.

Next we will consider a proper subset $S_{1}(F)$ of the singular set $S(F)$, which is open and closed in the relative topology and thus is analogous to a spectral set of a single operator. We will refer to such subsets as singular subsets.

THEOREM 1. Let $F(\lambda)$ be an operator valued function, analytic on its domain 4 , and such that $F(\lambda) F(\mu)=F(\mu) F(\lambda)$ for all $\lambda, \mu \in \Delta$. Let $S_{1}(F)$ be a bounded singular subset and let $f(\lambda)$ and $g(\lambda)$ be complex valued functions, analytic on their domains $\Delta f$ and $\Delta g$ respectively, open sets such that $S_{1}(F) \subset \Delta f \cap \Delta g$. Let $D_{1}$ and $D_{2}$ be bounded Cauchy domains such that $D_{1} \cap S(F)=S_{1}(F), \bar{D}_{1} \subset D_{2}$ and $\bar{D}_{2} \subset \Delta f \cap \Delta g$. Define the operators:

$$
\begin{array}{rlrl}
F_{f} & =\frac{1}{2 \pi i} \oint_{\partial D_{1}} f(\lambda) F^{\prime}(\lambda) F(\lambda)^{-1} d \lambda \\
Q(\lambda, \mu) & =\frac{F(\lambda)-F(\mu)}{\lambda-\mu} & & \text { for } \lambda \neq \mu \\
& =F^{\prime}(\lambda) & & \text { for } \lambda=\mu
\end{array}
$$

and

$$
\begin{aligned}
K_{f g}= & \frac{1}{2 \pi i} \oint_{\partial D_{1}} g(\lambda) F^{\prime}(\lambda) F(\lambda)^{-1} \\
& \times\left[\frac{1}{2 \pi i} \oint_{\partial D_{2}} f(\mu)(\lambda-\mu) F(\mu)^{-1} \frac{d}{d \mu} Q(\lambda, \mu) d \mu\right] d \lambda .
\end{aligned}
$$

Then $F_{f g}=F_{f} F_{g}+K_{f g}$.

Proof.

$$
F(\mu)^{-1}-F(\lambda)^{-1}=(\lambda-\mu) Q(\lambda, \mu) F(\lambda)^{-1} F(\mu)^{-1}
$$

so that

$$
F^{\prime}(\lambda) F(\lambda)^{-1}=F^{\prime}(\lambda) F(\mu)^{-1}-(\lambda-\mu) Q(\lambda, \mu) F(\mu)^{-1} F^{\prime}(\lambda) F(\lambda)^{-1} .
$$

From Figure 1: 


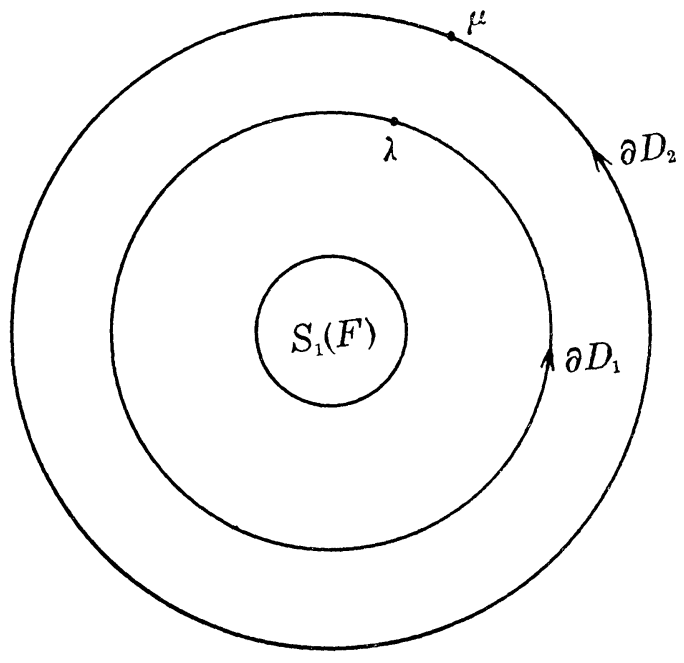

Figure 1

$$
\begin{aligned}
F_{f g}= & \frac{1}{2 \pi i} \oint_{\partial D_{1}} f(\lambda) g(\lambda) F^{\prime}(\lambda) F(\lambda)^{-1} d \lambda \\
= & \frac{1}{2 \pi i} \oint_{\partial D_{1}} g(\lambda)\left[\frac{1}{2 \pi i} \oint_{\partial D_{2}} \frac{f(\mu)}{\mu-\lambda} d \mu\right] F^{\prime}(\lambda) F(\lambda)^{-1} d \lambda \\
= & \frac{1}{2 \pi i} \oint_{\partial D_{1}} g(\lambda)\left\{\frac{1}{2 \pi i} \oint_{\partial D_{2}} \frac{f(\mu)}{\mu-\lambda}\right. \\
& \left.\quad \times\left[F^{\prime}(\lambda) F(\mu)^{-1}-(\lambda-\mu) Q(\lambda, \mu) F(\mu)^{-1} F^{\prime}(\lambda) F(\lambda)^{-1}\right] d \mu\right\} d \lambda \\
= & \frac{1}{2 \pi i} \oint_{\partial D_{2}} f(\mu) F(\mu)^{-1}\left[\frac{1}{2 \pi i} \oint_{\partial D_{1}} \frac{g(\lambda) F^{\prime}(\lambda)}{\mu-\lambda} d \lambda\right] d \mu \\
& +\frac{1}{2 \pi i} \oint_{\partial D_{1}} g(\lambda) F^{\prime}(\lambda) F(\lambda)^{-1}\left[\frac{1}{2 \pi i} \oint_{\partial D_{2}} f(\mu) Q(\lambda, \mu) F(\mu)^{-1} d \mu\right] d \lambda .
\end{aligned}
$$

The first integral on the right above is zero since $\mu \notin D_{1}$ and thus $\left(g(\lambda) F^{\prime}(\lambda)\right) /(\mu-\lambda)$ is analytic on $D_{1}$. Also

$$
Q(\lambda, \mu)=F^{\prime}(\mu)+(\lambda-\mu) \frac{d}{d \mu} Q(\lambda, \mu) \quad \text { so } \quad F_{f g}=F_{f} F_{g}+K_{f g} .
$$

We are concerned with singular subsets such that $K_{f g}=0$ for all such $f(\lambda)$ and $g(\lambda)$, in which case we obtain an algebraic homomorphism between analytic functions and operators similar to the case of spectral theory of a single operator.

Definition. Let $S_{1}(F)$ be a proper bounded singular subset of $S(F(\lambda))$ and let $Q(\lambda, \mu)$ (as defined in Theorem 1.) be invertible for all $\lambda, \mu \in S_{1}(F)$. Then $S_{1}(F)$ will be called separating. 
THEOREM 2. Let $S_{1}(F)$ be a bounded separating singular subset of $S(F(\lambda))$ and $D_{1}, D_{2}, f(\lambda), g(\lambda), F_{f}$ and $K_{f g}$ defined as in Theorem 1. Then $K_{f g}=0$ for all such $f(\lambda)$ and $g(\lambda)$.

Proof. First of all, it is clear that $Q(\lambda, \mu)$ is continuous at any $\lambda_{0}, \mu_{0}$ for $\lambda_{0} \neq \mu_{0}$ from the basic definition. If $\lambda_{0}=\mu_{0}$, then it is easy to see that $\left\|Q(\lambda, \mu)-F^{\prime}\left(\lambda_{0}\right)\right\| \rightarrow 0$ as $\lambda, \mu \rightarrow \lambda_{0}$ so that $Q(\lambda, \mu)$ is a continuous map on the product space $\mathscr{C} \times \mathscr{C}$ into $B(X)$, the space of bounded linear operators on $X$, where $\mathscr{C}$ is the complex plane. Also if $Q(\lambda, \mu)$ is invertible and if $\lambda^{\prime}, \mu^{\prime}$ are chosen so that $\left\|Q\left(\lambda^{\prime}, \mu^{\prime}\right)-Q(\lambda, \mu)\right\|<\left\|Q(\lambda, \mu)^{-1}\right\|^{-1}$, then $Q\left(\lambda^{\prime}, \mu^{\prime}\right)$ is also invertible. So the set of $Q(\lambda, \mu)$ 's which are invertible is an open set in the uniform topology of operators. Therefore the inverse image of the set of invertible $Q(\lambda, \mu)$ 's in $B(X)$, is an open set in $\mathscr{C} \times \mathscr{C}$. Thus $S_{1}(F)$ is contained in some open neighborhood $U$ upon which $Q(\lambda, \mu)$ is invertible. Since $\mathscr{C}$ is a Euclidean space, $D_{2}$ can be chosen so that $\partial D_{2}$ lies in $U \cap R(F)$. Hence on $D_{2}$ we can write $I=Q(\lambda, \mu) Q(\lambda, \mu)^{-1}$. As remarked above, $Q(\lambda, \mu)^{-1}$ is analytic on $D_{2}$.

$$
\begin{aligned}
K_{f g}= & \frac{1}{2 \pi i} \oint_{\partial D_{1}} g(\lambda) F^{\prime}(\lambda) F(\lambda)^{-1} \\
& \times\left[\frac{1}{2 \pi i} \oint_{\partial D_{2}} f(\mu)(\lambda-\mu) Q(\lambda, \mu) Q(\lambda, \mu)^{-1} F(\mu)^{-1} \frac{d}{d \mu} Q(\lambda, \mu) d \mu\right] d \lambda \\
= & \frac{1}{2 \pi i} \oint_{\partial D_{1}} g(\lambda) F^{\prime}(\lambda) F(\lambda)^{-1} \\
& \times\left[\frac{1}{2 \pi i} \oint_{\partial D_{2}} f(\mu)\left\{F(\lambda) F(\mu)^{-1}-I\right\} Q(\lambda, \mu)^{-1} \frac{d}{d \mu} Q(\lambda, \mu) d \mu\right] d \lambda \\
= & \frac{1}{2 \pi i} \oint_{\partial D_{2}} f(\mu) F(\mu)^{-1}\left[\frac{1}{2 \pi i} \oint_{\partial D_{1}} g(\lambda) F^{\prime}(\lambda) Q(\lambda, \mu)^{-1} \frac{d}{d \mu} Q(\lambda, \mu) d \lambda\right] d \mu \\
& -\frac{1}{2 \pi i} \oint_{\partial D_{1}} g(\lambda) F^{\prime}(\lambda) F(\lambda)^{-1}\left[\frac{1}{2 \pi i} \oint_{\partial D_{2}} f(\mu) Q(\lambda, \mu)^{-1} \frac{d}{d \mu} Q(\lambda, \mu) d \mu\right] d \lambda \\
= & 0
\end{aligned}
$$

since in each of the interior integrals, the integrands are analytic.

REMARK. While the condition $Q(\lambda, \mu)$ invertible on $S_{1}(F)$ is sufficient to insure that $K_{f g}=0$, examples can be easily constructed to show that this is not a necessary condition in all cases.

If $S_{1}(F)$ is a separating singular subset, let $f(\lambda) \equiv 1$. Then $F_{1}^{2}=F_{1} F_{1}=F_{1}$ by Theorem 2 . So defining

$$
P=F_{1}=\frac{1}{2 \pi i} \oint_{\partial D_{1}} F^{\prime}(\lambda) F(\lambda)^{-1} d \lambda
$$


$P$ is a projection. Let range $P=M$ and null space $P=N$. It is easy to give examples of a separating singular subset $S_{1}(F) \subset S(F)$ and yet where $M=X$. In general we cannot break $S(F)$ down into complementary separating sets, the ranges of whose projections provide a direct sum decomposition of $X$.

Corollary 3. $M$ is closed and $F_{f}$ is completely reduced by $M \oplus N$.

Proof. Since $P$ is a projection, $X=M \oplus N$. Since $F^{\prime}(\lambda) F(\lambda)^{-1}$ is bounded for $\lambda \in \partial D_{1}, P$ is the integral of a bounded operator valued function on a compact set, and hence $P$ is a bounded projection. Since domain $P=X, M$ is closed, a well known property of bounded projections. Also $F_{f} P=F_{f} F_{1}=F_{f}=F_{1} F_{f}=P F_{f}$, so $F_{f}$ is completely reduced by $M \oplus N$.

Let $\lambda_{0} \in R(F)$, then $F(\lambda)=\sum_{i=0}^{\infty} A_{j}\left(\lambda-\lambda_{0}\right)^{j}$ in some disc $C$ around $\lambda_{0}$ where as usual $A_{i}=(1 / 2 \pi i) \oint_{\partial C}\left(\lambda-\lambda_{0}\right)^{-j-1} F(\lambda) d \lambda$. The $A_{j}$ 's commute with $F(\lambda)$ and $F^{\prime}(\lambda)$ and hence also with $F(\lambda)^{-1}$. Thus the $A_{j}$ 's also commute with sums of $F^{\prime}(\lambda) F(\lambda)^{-1}$ and finally with uniform limits of such sums. So for each $j, A_{j} P=P A_{j}$ and these operator coefficients are also completely reduced by $M \oplus N$.

There are many interesting properties associated with spectral sets of a single operator. The next task is to see which of these will carry over to the separating singular subsets of $F(\lambda)$.

Lemima 4. Let $X=N \oplus R$, a Banach space and $B, A \in B(X)$. Assume $A$ is completely reduced by $N$ and $R$ and that $A \mid N$ is nilpotent and $A \mid R$ is one-to-one onto. Then if $A B=B A, B$ is also completely reduced by $N$ and $R$.

Proof. 0 is a pole of $(\lambda I-A)^{-1}$ and the projection

$$
P_{0}=\frac{1}{2 \pi i} \oint_{\Gamma}(\lambda I-A)^{-1} d \lambda
$$

has range $N$ and null space $R$, where $\Gamma$ is a contour in $\rho(A)$ enclosing only 0 of $\sigma(A)$. Since $B$ commutes with $A, B$ also commutes with $(\lambda I-A)^{-1}$ and with uniform limits of sums of such operators, so that $B P_{0}=P_{0} B$. Hence $B$ is completely reduced by $N$ and $R$.

Lemma 5. Let $\lambda_{0}$ be an isolated separating singular point such that ascent $F\left(\lambda_{0}\right)=$ descent $F\left(\lambda_{0}\right)=\nu<\infty$. Then $F^{\prime}(\lambda) F(\lambda)^{-1}$ has $a$ pole at $\lambda_{0}$ of order $\nu$. 
Proof. First of all, we recall that by definition $\nu$ is the smallest integer such that null space $F\left(\lambda_{0}\right)^{\nu}=$ null space $F\left(\lambda_{0}\right)^{\nu+1}$ and range $F\left(\lambda_{0}\right)^{\nu}=$ range $F\left(\lambda_{0}\right)^{\nu+1}$, and as is well known (e.g. see A. Taylor [4, p. 273]) $X=N\left(F\left(\lambda_{0}\right)^{\nu}\right) \oplus R\left(F\left(\lambda_{0}\right)^{\nu}\right)$ where $N$ means null space and $R$ means range. Also $F\left(\lambda_{0}\right)$ is completely reduced by this pair of subspaces and $F\left(\lambda_{0}\right)$ is continuously invertible on $R\left(F\left(\lambda_{0}\right)^{2}\right)$. In a neighborhood of $\lambda_{0}$ we can write:

$$
F(\lambda)=F^{\prime}\left(\lambda_{0}\right)+F^{\prime}\left(\lambda_{0}\right)\left(\lambda-\lambda_{0}\right)+\cdots+F^{(n)}\left(\lambda_{0}\right) \frac{\left(\lambda-\lambda_{0}\right)^{n}}{n !}+\cdots
$$

so that

$$
\begin{aligned}
& \left(\lambda-\lambda_{0}\right) Q\left(\lambda, \lambda_{0}\right) \\
& \quad=\left(\lambda-\lambda_{0}\right)\left[F^{\prime}\left(\lambda_{0}\right)+\cdots+F^{(n)}\left(\lambda_{0}\right) \frac{\left(\lambda-\lambda_{0}\right)^{n-1}}{n !}+\cdots\right] .
\end{aligned}
$$

Since $F(\lambda) F\left(\lambda_{0}\right)=F\left(\lambda_{0}\right) F(\lambda)$, by Lemma 4 , it is also true that $F(\lambda)$ is completely reduced by the pair of subspaces $N=N\left(F\left(\lambda_{0}\right)^{\nu}\right)$ and $R=R\left(F\left(\lambda_{0}\right)^{\nu}\right)$. So we can restrict $F(\lambda)$ to $N$ and write:

$$
-\left(\lambda_{0}-\lambda\right)^{\nu} Q\left(\lambda, \lambda_{0}\right)^{\nu}=F\left(\lambda_{0}\right)^{\nu}-\left(\lambda_{0}-\lambda\right)^{\nu} Q\left(\lambda, \lambda_{c}\right)^{\nu}
$$

since $F\left(\lambda_{0}\right)^{\nu} \mid N=0$, so that on $N$ :

$$
\begin{aligned}
& -\left(\lambda_{0}-\lambda\right)^{\nu} Q\left(\lambda, \lambda_{0}\right)^{\nu}=\left[F\left(\lambda_{0}\right)-\left(\lambda_{0}-\lambda\right) Q\left(\lambda, \lambda_{0}\right)\right] . \\
& \quad \times\left[F\left(\lambda_{0}\right)^{\nu-1}+F\left(\lambda_{0}\right)^{\nu-2}\left(\lambda_{0}-\lambda\right) Q\left(\lambda, \lambda_{0}\right)+\cdots+\left(\lambda_{0}-\lambda\right)^{\nu-1} Q\left(\lambda, \lambda_{0}\right)^{\nu-1}\right] .
\end{aligned}
$$

By definition $F(\lambda)=F\left(\lambda_{0}\right)-\left(\lambda_{0}-\lambda\right) Q\left(\lambda, \lambda_{0}\right)$ so that on $N$ :

$$
\begin{aligned}
& -F(\lambda)^{-1}\left(\lambda_{0}-\lambda\right)^{\nu} Q\left(\lambda, \lambda_{0}\right)^{\nu} \\
& \quad=F\left(\lambda_{0}\right)^{\nu-1}+\cdots+\left(\lambda_{0}-\lambda\right)^{\nu-1} Q\left(\lambda, \lambda_{0}\right)^{\nu-1} .
\end{aligned}
$$

Now since

$$
Q\left(\lambda, \lambda_{0}\right)=F^{\prime}\left(\lambda_{0}\right)+F^{\prime \prime}\left(\lambda_{0}\right) \frac{\left(\lambda-\lambda_{0}\right)}{2 !}+\cdots+F^{(n)}\left(\lambda_{0}\right) \frac{\left(\lambda-\lambda_{0}\right)^{n-1}}{n !}+\cdots
$$

and since $F^{\prime}\left(\lambda_{0}\right) \mid N$ is invertible because $\lambda_{0}$ is a separating singular point, it is also true that there is some $\rho>0$ such that $\left|\lambda-\lambda_{0}\right|<\rho$ implies that $Q\left(\lambda, \lambda_{0}\right) \mid N$ is also invertible. Therefore on $N$ :

$$
-F(\lambda)^{-1}=\frac{F\left(\lambda_{0}\right)^{\nu-1} Q\left(\lambda, \lambda_{0}\right)^{-\nu}}{\left(\lambda_{0}-\lambda\right)^{\nu}}+\cdots+\frac{Q\left(\lambda, \lambda_{0}\right)}{\lambda_{0}-\lambda} .
$$

Since $F(\lambda)=F\left(\lambda_{0}\right)+\left(\lambda-\lambda_{0}\right) Q\left(\lambda, \lambda_{0}\right)$, we have:

$$
F^{\prime}(\lambda)=Q\left(\lambda, \lambda_{0}\right)-\left(\lambda_{0}-\lambda\right) Q^{\prime}\left(\lambda, \lambda_{0}\right)
$$


which we apply to both sides of equation (3)

$$
\begin{aligned}
&-F^{\prime}(\lambda) F(\lambda)^{-1}= {\left[Q\left(\lambda, \lambda_{0}\right)-\left(\lambda_{0}-\lambda\right) Q^{\prime}\left(\lambda, \lambda_{0}\right)\right] } \\
& \times\left[\frac{F\left(\lambda_{0}\right)^{\nu-1} Q\left(\lambda, \lambda_{0}\right)^{-\nu}}{\left(\lambda_{0}-\lambda\right)^{\nu}}+\cdots+\frac{Q\left(\lambda, \lambda_{0}\right)}{\lambda_{0}-\lambda}\right] \\
&=\sum_{k=0}^{\nu-1} F\left(\lambda_{0}\right)^{k}\left[\frac{Q\left(\lambda, \lambda_{0}\right)^{-k}-\left(\lambda_{0}-\lambda\right) Q\left(\lambda, \lambda_{0}\right)^{-k-1} Q^{\prime}\left(\lambda, \lambda_{0}\right)}{\left(\lambda_{0}-\lambda\right)^{k+1}}\right] .
\end{aligned}
$$

From equation (2)

$$
Q\left(\lambda, \lambda_{0}\right)^{-1}=\left(F^{\prime}\left(\lambda_{0}\right)\right)^{-1}\left[I+\left(F^{\prime}\left(\lambda_{0}\right)\right)^{-1} \sum_{k=2}^{\infty} \frac{F^{(k)}\left(\lambda_{0}\right)\left(\lambda-\lambda_{0}\right)^{k-1}}{k !}\right]^{-1}
$$

so for $\left|\lambda-\lambda_{0}\right|$ sufficiently small, $Q\left(\lambda, \lambda_{0}\right)^{-1}$ has a Neumann expansion in positive powers of $\left(\lambda_{0}-\lambda\right)$. Also from equation (2) $Q^{\prime}\left(\lambda, \lambda_{0}\right)$ has only nonnegative powers of $\left(\lambda_{0}-\lambda\right)$, so that in equation (4), $F^{\prime}(\lambda) F(\lambda)^{-1} \mid N$ has $\nu$ as the highest negative power of $\left(\lambda_{0}-\lambda\right)$.

Next we consider the restriction to the complementary subspace R. $F\left(\lambda_{0}\right) \mid R$ is invertible and $F(\lambda)-F\left(\lambda_{0}\right)=\left(\lambda-\lambda_{0}\right) Q\left(\lambda, \lambda_{0}\right)$. So for $\left|\lambda-\lambda_{0}\right|$ sufficiently small $\left\|F(\lambda)-F\left(\lambda_{0}\right)\right\| \leqq\left\|F\left(\lambda_{0}\right)^{-1}\right\|^{-1}$. Thus by a well known theorem (e.g. see Dunford and Schwartz [1, p. 584]) $F(\lambda) \mid R$ is also invertible and restricted to $R$ :

$$
\begin{aligned}
F(\lambda)^{-1} & =F\left(\lambda_{0}\right)^{-1} \sum_{k=0}^{\infty}\left\{\left[F(\lambda)-F\left(\lambda_{0}\right)\right] F\left(\lambda_{0}\right)^{-1}\right\}^{k} \\
& =F\left(\lambda_{0}\right)^{-1} \sum_{k=0}^{\infty}\left\{\left(\lambda-\lambda_{0}\right) Q\left(\lambda, \lambda_{0}\right) F\left(\lambda_{0}\right)^{-1}\right\}^{k}
\end{aligned}
$$

which has only nonnegative powers of $\left(\lambda-\lambda_{0}\right)$. Thus on $X=N \oplus R$ we can write:

$$
\begin{aligned}
F^{\prime}(\lambda) F(\lambda)^{-1} & \\
= & \left\{-\sum_{k=1}^{\nu-1} \frac{F\left(\lambda_{0}\right)^{k}}{k} \frac{d}{d \lambda}\left[\frac{Q\left(\lambda, \lambda_{0}\right)^{-k}}{\left(\lambda_{0}-\lambda\right)^{k}}\right]+\frac{I}{\lambda_{0}-\lambda}-Q\left(\lambda, \lambda_{0}\right)^{-1} Q^{\prime}\left(\lambda, \lambda_{0}\right)\right\} \\
& \oplus\left\{F^{\prime}(\lambda) F\left(\lambda_{0}\right)^{-1} \sum_{k=0}^{\infty}\left[\left(\lambda-\lambda_{0}\right) Q\left(\lambda, \lambda_{0}\right) F\left(\lambda_{0}\right)^{-1}\right]^{k}\right\}
\end{aligned}
$$

and so $F^{\prime}(\lambda) F(\lambda)^{-1}$ has a pole of order $\nu$ at $\lambda_{0}$.

LEMMA 6. Let ascent $F\left(\lambda_{0}\right)=$ descent $F\left(\lambda_{0}\right)=\nu<\infty$ and assume $F^{\prime}\left(\lambda_{0}\right)$ is invertible. Then $\lambda_{0}$ is an isolated separating singular point.

Proof. The idea is based upon a proof of D. Lay [2] for a similar proposition for a point in the spectrum of a single operator. In the terminology of Lemma $5, F\left(\lambda_{0}\right) \mid N$ is nilpotent and also as in Lemma $5, Q\left(\lambda, \lambda_{0}\right)$ is invertible for $\left|\lambda-\lambda_{0}\right|$ sufficiently small. So 


$$
F(\lambda)\left|N=F\left(\lambda_{0}\right)\right| N+\left(\lambda-\lambda_{0}\right) Q\left(\lambda, \lambda_{0}\right) \mid N
$$

is the sum of a nilpotent operator and an invertible operator.

$$
\left(\lambda-\lambda_{0}\right)^{-1} Q\left(\lambda, \lambda_{0}\right)^{-1} F(\lambda)|N=I| N+\left(\lambda-\lambda_{0}\right)^{-1} Q\left(\lambda, \lambda_{0}\right)^{-1} F\left(\lambda_{0}\right) \mid N .
$$

The second term on the right is also nilpotent and hence has spectrum $=\{0\}$. Thus the right side of the above equation is invertible and hence $F(\lambda) \mid N$ is also invertible for $0<\left|\lambda-\lambda_{0}\right|<\varepsilon$ for some $\varepsilon>0$. On the complementary subspace $F\left(\lambda_{0}\right) \mid R$ is invertible and so $F(\lambda) \mid R$ is also invertible for $\left|\lambda-\lambda_{0}\right|$ sufficiently small. So on the entire space $X, F(\lambda)$ is invertible for $0<\left|\lambda-\lambda_{0}\right|<\varepsilon$. Therefore $\lambda_{0}$ is an isolated point of $S(F)$ and is separating since $F^{\prime}\left(\lambda_{0}\right)$ is invertible.

THEOREM 7. If ascent $F\left(\lambda_{0}\right)=$ descent $F\left(\lambda_{0}\right)=\nu<\infty$ and $F^{\prime \prime}\left(\lambda_{0}\right)$ is invertible, then $F^{\prime}(\lambda) F(\lambda)^{-1}$ has a pole of order $\nu$ at $\lambda_{0}$.

Proof. Combine Lemmas 5 and 6.

Before proceeding further, we define a new operator which will prove to be useful.

DEFINITION. Let $S_{1}(F)$ be a separating singular subset of $F(\lambda)$. Define

$$
T=\frac{1}{2 \pi i} \oint_{\partial D} \lambda F^{\prime}(\lambda) F(\lambda)^{-1} d \lambda
$$

where $\bar{D} \cap S(F)=S_{1}(F)$. $\quad T$ will be called the root operator associated with $F(\lambda)$ and $S_{1}\left(F^{\prime}\right)$. When it is not otherwise clear from the context, we will use the notation $T=T\left[F(\lambda), S_{1}(F)\right]$.

$T$ is a bounded operator on $X$ into $M$ where $M=$ range $P$ and $P$ is the projection associated with $S_{1}(F)$. The boundedness results from the fact that $|\lambda|$ and $\left\|F^{\prime}(\lambda) F(\lambda)^{-1}\right\|$ are bounded on $\partial D$, a compact set in $R(F)$. From the operational calculus:

$$
T=P T=T P
$$

so $T$ is completely reduced by $M$ and $N$ where $X=M \oplus N$ and $T \mid N=0$. From the definition of $f(\lambda)$ and $F_{f}$ in Theorem 1, let $f(\lambda)=k \lambda^{n}$. Then

$$
k T^{n}=\frac{1}{2 \pi i} \oint_{\partial D} k \lambda^{n} F^{\prime}(\lambda) F(\lambda)^{-1} d \lambda .
$$

So for $f(\lambda)$ a polynomial, $F_{f}=f(T)$. More generally, for $f(\lambda)$ any analytic function with domain as in Theorem 1, we may define 


$$
f(T)=\frac{1}{2 \pi i} \oint_{\partial D} f(\lambda) F^{\prime}(\lambda) F(\lambda)^{-1} d \lambda
$$

as is done for the case of a single bounded operator in reference [4] and elsewhere. Also since $F^{\prime}(\lambda) F(\lambda)^{-1}$ is bounded on $\partial D$, if $f_{n}(\lambda) \rightarrow$ $f(\lambda)$ uniformly on a compact set containing $S_{1}(F), f_{n}(T) \rightarrow f(T)$ in norm. We could also consider $f(T)$ as the limit of $f_{n}(T)$, polynomials in $T$.

Upon reviewing the proof of Theorem 1, we observe that the complex valued analytic functions $f(\lambda)$ and $g(\lambda)$ could just as well have been replaced by vector valued functions with appropriate domains of analyticity, provided that these vector valued functions commute with $F(\lambda)$.

Definition. Let $S_{1}(F)$ be a separating singular subset for $F(\lambda)$ and $T=T\left[S_{1}(F), F(\lambda)\right]$ be the associated root operator. If $G(\lambda)$ is an operator valued function, analytic on $D$ where $D \cap S(F)=S_{1}(F)$, and such that $G(\lambda) F(\lambda)=F(\lambda) G(\lambda)$ for all $\lambda \in D$, we define:

$$
G(T)=\frac{1}{2 \pi i} \oint_{\partial D} G(\lambda) F^{\prime}(\lambda) F(\lambda)^{-1} d \lambda .
$$

THEOREM 8. Let $S_{1}(F)$ be a separating singular subset and $T$ the associated root operator. Then $F(T)=0$.

Proof.

$$
F(T)=\frac{1}{2 \pi i} \oint_{\partial D} F(\lambda) F^{\prime}(\lambda) F(\lambda)^{-1} d \lambda=\frac{1}{2 \pi i} \oint_{\partial D} F^{\prime}(\lambda) d \lambda=0
$$

since $F^{\prime}(\lambda)$ is analytic on $D$.

THEOREM 9. Let $S_{1}(F)$ be a separating singular subset and $T=$ $T\left[S_{1}(F), F(\lambda)\right]$, the root operator, and $M=$ range $P$ where $P$ is the projection corresponding to $S_{1}(F)$. Then $\sigma(T \mid M)=S_{1}(F)$.

Proof. Suppose $a \notin S_{1}(F)$ but $a \in \sigma(T \mid M)$. We can then choose a contour $\Gamma$ around $S_{1}(F)$ so that $a$ is outside of $\Gamma$. Then since $a \neq \lambda$ on or inside of $\Gamma,(a-\lambda)^{-1}$ is analytic on and inside of $\Gamma$. Let

$$
G=\frac{1}{2 \pi i} \oint_{\Gamma}(a-\lambda)^{-1} F^{\prime}(\lambda) F(\lambda)^{-1} d \lambda .
$$

Then since

$$
(a P-T)=\frac{1}{2 \pi i} \oint_{\Gamma}(a-\lambda) F^{\prime}(\lambda) F(\lambda)^{-1} d \lambda
$$


and making use of the operational calculus:

$$
G(a P-T)=(a P-T) G=P
$$

so that $G=(\alpha P-T)^{-1}$ on $M$, a contradiction since $a \in \sigma(T \mid M)$. Therefore $a \notin S_{1}(F)$ implies $a \in \rho(T \mid M)$ or $\sigma(T \mid M) \subseteq S_{1}(F)$. Next assume $a \in S_{1}(F)$. As shown in the proof of Theorem 2, $Q(\lambda, \mu)$ is invertible on $U$, some open set such that $S_{1}(F) \subset U$. Choose $\lambda \in U \cap R(F)$. Then $F(a)$ is not invertible but $F(\lambda)$ is, and

$$
Q(a, \lambda)=\frac{F(a)-F(\lambda)}{a-\lambda}
$$

is invertible also. So

$$
[F(a)-F(\lambda)] Q(a, \lambda)^{-1}=(a-\lambda) I .
$$

Applying the integral operator to both sides with $\bar{D} \subset U$ and recalling that $Q(a, \lambda)^{-1}$ is analytic on $U$ :

$$
\begin{gathered}
\frac{1}{2 \pi i} \oint_{\partial D} F(a) Q(a, \lambda)^{-1} F^{\prime}(\lambda) F(\lambda)^{-1} d \lambda-\frac{1}{2 \pi i} \oint_{\partial D} F(\lambda) Q(a, \lambda)^{-1} F^{\prime}(\lambda) F(\lambda)^{-1} d \lambda \\
=\frac{1}{2 \pi i} \oint_{\partial D}(a-\lambda) F^{\prime}(\lambda) F(\lambda)^{-1} d \lambda .
\end{gathered}
$$

The second integral on the left is zero since the integrand in analytic on $\bar{D}$. So we obtain:

$$
F(a) P Q(a, T)^{-1}=(a P-T)
$$

so that $F(a)$ not invertible implies $(a P-T)$ not invertible. Hence $S_{1}(F) \subseteq \sigma(T \mid M)$ and $S_{1}(F)=\sigma(T \mid M)$.

REMARK. Since $T \mid N=0$, we note that $S_{1}(F) \subseteq \sigma(T)$ and if $0 \in S_{1}(F)$, then $S_{1}(F)=\sigma(T)$.

Now $M$ being a closed subspace of $X$ may be considered as a Banach space in its own right. So we may consider the usual properties of the resolvent operator involving $T \mid M$. So since $\sigma(T \mid M)=S_{1}(F)$ :

$$
I\left|M=\frac{1}{2 \pi i} \oint_{\partial D}(\lambda I-T)^{-1} d \lambda\right| M=P
$$

where $P$ is the projection obtained from $F^{\prime}(\lambda) F(\lambda)^{-1}$. More generally, for any polynomial $f_{n}(\lambda)$ :

$$
\frac{1}{2 \pi i} \oint_{\partial D} f_{n}(\lambda)(\lambda P-T)^{-1} d \lambda=f_{n}(T)=\frac{1}{2 \pi i} \oint_{\partial D} f_{n}(\lambda) F^{\prime}(\lambda) F(\lambda)^{-1} d \lambda .
$$


Note that we can drop the restriction to $M$, since on $N$, both sides will be zero. As before if $f_{n}(\lambda) \rightarrow f(\lambda)$ uniformly on a compact set containing $S_{1}(F)$, then $f_{n}(T) \rightarrow f(T)$ in norm, and the limit of the operators defined by each integral must be the same.

CoRollary 10. Let $S_{1}(F)$ be a separating singular subset and $f(\lambda)$ analytic on an open set containing $D$, a bounded Cauchy domain such that $D \cap S(F)=S_{1}(F)$. Then $\sigma\left(F_{f} \mid M\right)=f\left(S_{1}(F)\right)$.

Proof. From the properties of the root operator $T$ associated with $F(\lambda)$ and $S_{1}(F)$ :

$$
F_{f}=\frac{1}{2 \pi i} \oint_{\partial D} f(\lambda) F^{\prime}(\lambda) F(\lambda)^{-1} d \lambda=f(T) .
$$

From the spectral mapping theorem $\sigma[f(T)]=f[\sigma(T)]$ so that

$$
\sigma\left(F_{f} \mid M\right)=\sigma[f(T \mid M)]=f(\sigma(T \mid M))=f\left(S_{1}(F)\right) .
$$

Next we can use the root operator $T$ to obtain a partial converse to Theorem 7. First as a lemma, we use a well known theorem.

Lemma 11. If $B$ is a bounded linear operator on a Banach space $X$, and if $\lambda_{0}$ is a pole of $(\lambda I-B)^{-1}$ of order $m$, then ascent $\left(\lambda_{0} I-B\right)=$ descent $\left(\lambda_{0} I-B\right)=m$ and $X=R\left[\left(\lambda_{0} I-B\right)^{m}\right] \oplus N\left[\left(\lambda_{0} I-B\right)^{m}\right]$. Further $N\left[\left(\lambda_{0} I-B\right)^{m}\right]$ is the range of the corresponding spectral projection.

Proof. See Taylor $[4$, p. 306].

LEMMA 12. Let $\lambda_{0} \neq 0$ be an isolated separating singular point for $F(\lambda)$ and let $M$ and $N$ be respectively the range and null space of the corresponding projection $P$. Then $F\left(\lambda_{0}\right) \mid M$ is not invertible and $F\left(\lambda_{0}\right) \mid N$ is continuously invertible.

Proof. From Corollary 3 and the remarks following, we know that $X=M \oplus N$ and $F\left(\lambda_{0}\right)$ is completely reduced by this pair of subspaces. If $F\left(\lambda_{0}\right) \mid M$ were invertible, then $F(\lambda) \mid M$ would be invertible in some open neighborhood of $\lambda_{0}$ and in this neighborhood $F(\lambda)^{-1} \mid M$ would be analytic. We would then have

$$
P=\frac{1}{2 \pi i} \oint_{\partial D} F^{\prime}(\lambda) F(\lambda)^{-1} d \lambda=0
$$

a contradiction.

Now suppose $F\left(\lambda_{0}\right) \mid N$ is not invertible and write $\hat{F}(\lambda)=F(\lambda) \mid N$. 
Then $\lambda_{0} \in S(\hat{F})$ and $\lambda_{0} \in R\left(\hat{F}^{\prime}\right)$ since $\lambda_{0} \in R\left(F^{\prime}\right)$, and $F^{\prime}(\lambda)$ is completely reduced by $M$ and $N$.

$$
\hat{T}=T \mid N=\frac{1}{2 \pi i} \oint_{\partial D} \lambda \hat{F}^{\prime}(\lambda) \hat{F}(\lambda)^{-1} d \lambda .
$$

By the remark after the definition of the root operator, $\widehat{T}=0$. Also by the remark after Theorem $9, \lambda_{0}=S_{1}(\hat{F}) \subseteq \sigma(\hat{T})=\{0\}$, a contradiction. So $\lambda_{0} \in R(\hat{F})$ and $\hat{F}\left(\lambda_{0}\right)$ is continuously invertible.

THEOREM 13. Let $\lambda_{0} \neq 0$ be an isolated separating singular point of $F(\lambda)$ and suppose $\lambda_{0}$ is a pole of $F^{\prime}(\lambda) F(\lambda)^{-1}$ of order $m$. Then ascent $F\left(\lambda_{0}\right)=$ descent $F\left(\lambda_{0}\right)=m$ and $X=R\left[F\left(\lambda_{0}\right)^{m}\right] \oplus N\left[F\left(\lambda_{0}\right)^{m}\right]$.

Proof. By Lemma 12, $F\left(\lambda_{0}\right) \mid M$ is not invertible and $F\left(\lambda_{0}\right) \mid N$ is continuously invertible. So

$$
N\left(F\left(\lambda_{0}\right)\right) \subseteq N\left(F\left(\lambda_{0}\right)^{2}\right) \subseteq \cdots \sqsubseteq N\left(F\left(\lambda_{0}\right)^{m}\right) \subseteq \cdots \sqsubseteq M
$$

and

$$
R\left(F\left(\lambda_{0}\right)\right) \supseteqq R\left(F\left(\lambda_{0}\right)^{2}\right) \supseteqq \cdots \supseteqq R\left(F\left(\lambda_{0}\right)^{m}\right) \supseteqq \cdots \supseteqq N
$$

In some neighborhood of $\lambda_{0}$, i.e. $\left|\lambda-\lambda_{0}\right|\langle\rho, \rho\rangle 0$, we can write:

$$
F^{\prime}(\lambda) F(\lambda)^{-1}=\sum_{n=0}^{\infty}\left(\lambda-\lambda_{0}\right)^{n} E_{n}+\sum_{n=1}^{m}\left(\lambda-\lambda_{0}\right)^{-n} F_{n}
$$

and $F_{n} \mid N=0$ for all $n$ since $F\left(\lambda_{\jmath}\right) \mid N$ is invertible. Also since $\left\{\lambda_{0}\right\}=\sigma(T \mid M)$, on $M$ we have:

$$
(\lambda I-T)^{-1}=\sum_{n=0}^{\infty}\left(\lambda-\lambda_{0}\right)^{n} C_{n}+\sum_{n=1}^{\infty}\left(\lambda-\lambda_{0}\right)^{-n} D_{n} .
$$

By the remarks after Theorem 9:

$$
\begin{aligned}
D_{n}=\frac{1}{2 \pi i} & \oint_{\partial D}\left(\lambda-\lambda_{0}\right)^{n-1}(\lambda I-T)^{-1} d \lambda \\
& =\frac{1}{2 \pi i} \oint_{\partial D}\left(\lambda-\lambda_{0}\right)^{n-1} F^{\prime}(\lambda) F(\lambda)^{-1} d \lambda=F_{n} .
\end{aligned}
$$

So $D_{n}=0$ for $n>m$ and thus $(\lambda I-T)^{-1}$ also has a pole of order $m$ at $\lambda_{0}$. So by Lemma 11, ascent $\left(\lambda_{0} I-T\right)=\operatorname{descent}\left(\lambda_{0} I-T\right)=m$ and $M=N\left[\left(\lambda_{0} I-T\right)^{m}\right]$. We can write $(\lambda I-T)=\left(\lambda I-\lambda_{0} I+\lambda_{0} I-T\right)$ and for $\lambda \neq \lambda_{0}\left|\lambda-\lambda_{0}\right|>r_{o}\left(\lambda_{0} I-T\right)=\lim _{n \rightarrow \infty}\left\|\left(\lambda_{0} I-T\right)^{n}\right\|^{1 / n}=0$ where $r_{\sigma}(T)=\sup _{\lambda_{\epsilon}(T)}|\lambda|$ is the spectral radius of an operator $T$. So

$$
(\lambda I-T)^{-1}=\sum_{n=1}^{\infty}\left(\lambda-\lambda_{0}\right)^{-n}\left(T-\lambda_{0} I\right)^{n-1},
$$


the Neumann expansion. This implies that $C_{n}=0$ for all $n$. So on $M$ we have:

$$
(\lambda P-T)^{-1}=\sum_{n=1}^{m}\left(\lambda-\lambda_{0}\right)^{-n} D_{n}=\sum_{n=1}^{m}\left(\lambda-\lambda_{0}\right)^{-n} F_{n},
$$

so that:

$$
F^{\prime}(\lambda) F(\lambda)^{-1}=\sum_{n=0}^{\infty}\left(\lambda-\lambda_{0}\right)^{n} E_{n}+(\lambda P-T)^{-1} .
$$

As was to be expected since $F\left(\lambda_{0}\right) \mid N$ is invertible, $F^{\prime}(\lambda) F(\lambda)^{-1} \mid N$ has a Taylor series expansion around $\lambda_{0}$. Taking advantage of commutativity and rearranging terms:

$$
F^{\prime}(\lambda)(\lambda P-T)=F(\lambda)\left[\sum_{n=0}^{\infty}\left(\lambda-\lambda_{0}\right)^{n} E_{n}(\lambda P-T)+P\right]
$$

SO

$$
F^{\prime}\left(\lambda_{0}\right)\left(\lambda_{0} P-T\right)=F\left(\lambda_{0}\right)\left[E_{0}\left(\lambda_{0} P-T\right)+P\right] .
$$

On $M,\left(\lambda_{0} P-T\right)$ is nilpotent. Hence $E_{0}\left(\lambda_{0} P-T\right)+P$ is invertible. $F^{\prime}\left(\lambda_{0}\right)$ is also invertible since $\lambda_{0}$ is separating. Therefore $\left(\lambda_{0} P-T\right)^{m}=0$ implies that $F\left(\lambda_{0}\right)^{m} \mid M=0$ and $N\left[F\left(\lambda_{0}\right)^{m}\right]=N\left[\left(\lambda_{0} P-T\right)^{m}\right]=M$. Also on $M: R\left[F\left(\lambda_{0}\right)^{m}\right]=R\left[\left(\lambda_{0} P-T\right)^{m}\right]=(0)$. On $\left.N: R\left(F\left(\lambda_{0}\right)\right)\right)=N=$ $R\left[F\left(\lambda_{0}\right)^{n}\right]$ for all $n$ since $F\left(\lambda_{0}\right) \mid N$ is 1 to 1 . So on $X=$ $M \oplus N R\left[F\left(\lambda_{0}\right)^{m}\right]=R\left[F\left(\lambda_{0}\right)^{m+q}\right]=(0) \cup N=N$ for all $q \geqq 0$. So ascent $F\left(\lambda_{0}\right)=$ descent $F\left(\lambda_{0}\right)=m$ and $X=N\left[F\left(\lambda_{0}\right)^{m}\right] \oplus R\left[F\left(\lambda_{0}\right)^{m}\right]$.

COROLlaRY 14. Let $\lambda_{0} \neq 0$ be an isolated separating singular point of $F(\lambda)$. Let $P$ be the corresponding projection and $M=$ range $P$. Then $M$ is characterized by $M=\left\{x \mid\left\|F\left(\lambda_{0}\right)^{n}\right\|^{1 / n} \rightarrow 0\right\}$.

Proof. Equations (5) and (6) hold for $\lambda_{0}$ any isolated separating singular point as no assumptions about ascent and descent were needed to obtain them. So again $F^{\prime \prime}\left(\lambda_{0}\right)\left(\lambda_{0} P-T\right)=F\left(\lambda_{0}\right)\left[E_{0}\left(\lambda_{0} P-T\right)+P\right]$. Since $\sigma(T \mid M)=\left\{\lambda_{0}\right\},\left(\lambda_{0} P-T\right) \mid M$ is quasinilpotent. Therefore $E_{0}\left(\lambda_{0} P-T\right)+P$ is invertible on $M$. So

$$
F\left(\lambda_{0}\right)\left|M=F^{\prime}\left(\lambda_{0}\right)\left(\lambda_{0} P-T\right)\left[E_{0}\left(\lambda_{0} P-T\right)+P\right]^{-1}\right| M
$$

is also quasinilpotent, and as before $F\left(\lambda_{0}\right) \mid N$ is invertible.

\section{REFERENCES}

1. N. Dunford and J. T. Schwartz, Linear Operators Vol $I$, Interscience, New York, 1964.

2. David Lay, Studies in Ascent, Descent, and Nullity of Closed Operators, Ph. D. 
Dissertation, University of California, Los Angeles, 1966.

3. Angus E. Taylor, Linear operators which depend analytically on a parameter, Ann. of Math. 39 (1938), 574-593.

4. — Introduction to Functional Analysis, Wiley and Sons, New York, 1958.

Received November 22, 1966. The results presented in this paper are a part of the author's Ph. D. dissertation at UCLA, written under the direction of Professor Angus E. Taylor.

United States Army Research and Development Group

FRANKFURT, Germany 


\section{PACIFIC JOURNAL OF MATHEMATICS}

\section{EDITORS}

\section{H. ROYDEN}

Stanford University

Stanford, California

\section{J. P. Jans}

University of Washington

Seattle, Washington 98105

\section{J. DUGUNDJI}

Department of Mathematics

Rice University

Houston, Texas 77001

RICHARD ARENS

University of California

Los Angeles, California 90024

\section{ASSOCIATE EDITORS}
E. F. BeCKenbaCH
B. H. NeumanN
F. WOLF
K. YosIDA

\section{SUPPORTING INSTITUTIONS}

UNIVERSITY OF BRITISH COLUMBIA CALIFORNIA INSTITUTE OF TECHNOLOGY UNIVERSITY OF CALIFORNIA MONTANA STATE UNIVERSITY UNIVERSITY OF NEVADA NEW MEXICO STATE UNIVERSITY OREGON STATE UNIVERSITY UNIVERSITY OF OREGON OSAKA UNIVERSITY UNIVERSITY OF SOUTHERN CALIFORNIA
STANFORD UNIVERSITY UNIVERSITY OF TOKYO UNIVERSITY OF UTAH WASHINGTON STATE UNIVERSITY UNIVERSITY OF WASHINGTON

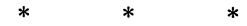

AMERICAN MATHEMATICAL SOCIETY CHEVRON RESEARCH CORPORATION TRW SYSTEMS

NAVAL ORDNANCE TEST STATION 


\section{Pacific Journal of Mathematics}

\section{Vol. 24, No. $1 \quad$ May, 1968}

Harry P. Allen, Lie algebras of type $D_{4}$ over algebraic number fields ...... 1

Charles Ballantine, Products of positive definite matrices. II............ 7

David W. Boyd, The spectral radius of averaging operators ............ 19

William Howard Caldwell, Hypercyclic rings ................... 29

Francis William Carroll, Some properties of sequences, with an application

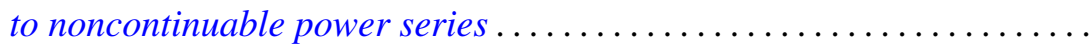

David Fleming Dawson, Matrix summability over certain classes of

sequences ordered with respect to rate of convergence ........... 51

D. W. Dubois, Second note on David Harrison's theory of preprimes. . . . . 57

Edgar Earle Enochs, A note on quasi-Frobenius rings.............. 69

Ronald J. Ensey, Isomorphism invariants for Abelian groups modulo bounded groups ................................ 71

Ronald Owen Fulp, Generalized semigroup kernels ................ 93

Bernard Robert Kripke and Richard Bruce Holmes, Interposition and approximation ................................. 103

Jack W. Macki and James Sai-Wing Wong, Oscillation of solutions to second-order nonlinear differential equations ..................

Lothrop Mittenthal, Operator valued analytic functions and generalizations

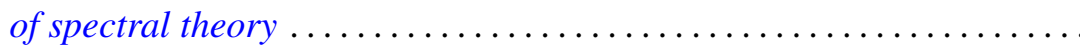

T. S. Motzkin and J. L. Walsh, A persistent local maximum of the pth power deviation on an interval, $p<1 \ldots \ldots \ldots \ldots \ldots \ldots \ldots \ldots \ldots \ldots . \ldots \ldots$

Jerome L. Paul, Sequences of homeomorphisms which converge to homeomorphisms ...........................

Maxwell Alexander Rosenlicht, Liouville's theorem on functions with elementary integrals.

Joseph Goeffrey Rosenstein, Initial segments of degrees .

$\mathrm{H}$. Subramanian, Ideal neighbourhoods in a ring ............

Dalton Tarwater, Galois cohomology of abelian groups . .

James Patrick Williams, Schwarz norms for operators ... .

Raymond Y. T. Wong, A wild Cantor set in the Hilbert cube. 